\title{
MERCURY IN SHALLOW SAVANNAH RIVER PLANT SOIL
}

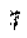

Published: October 1988

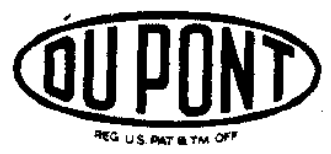

E. I. du Pont de Nemours \& Co. Savannah River Laboratory Aiken, SC 29808 


\section{DISCLAMMER}

This report was prepared by E. I. du Pont de Nemours and Company (DuPont) for the United States Department of Energy under Contract DE-AC09-76SR00001 and is an account of work performed under that Contract. Nelther the United States, the United Stated Department of Energy nor Du Pont, nor any of their omployees, makes any warranty, express or implied, or assumes any legal liability or responsiblitty for the accuracy, completeness, or usefuliness of any informiation, apparatus, product, or process dlsclosed herein, or represents that lis use will not Infringe provately owned rights. Reference herein to any specific commerclal product, process or service by trade name, mark, manufacturer, or otherwise does not necessarily constitute or imply endorsement, recommendation, or favoring of same by Du Pont or by the Untted States Government or any agency thereof. The views and opinions of authors expressed herein do not necessarlly state or reflect those of the United States Government or any agency thereof. 
Key Words: Mercury

\section{MERCURY IN SHALLOW SAVANNAH RIVER PLANT SOIL}

by

V. Price

J. R. Cook

Approved by:

J. C. Corey, Research Manager

Published: October 1988

E. I. du Pont de Nemours \& Co. Savannah River Laboratory Aiken, SC 29808 PREPARED FOR THE U.S. DEPARTMENT OF ENERGY UNDER CONTRACT DE-ACO9-76SRO0001 
TABLE OF CONTENTS

$\begin{array}{lr}\text { List of Tables } & \text { ii } \\ \text { List of Figures } & \text { iv } \\ \text { Executive Summary } & 1 \\ \text { Introduction } & 1 \\ \text { Sampling Areas } & 2 \\ \text { Sample Collection } & 2 \\ \text { Sample Analysis } & - \\ \text { Analytical Results } & 2 \\ \text { References } & 3\end{array}$

Page

ii i

iv

1

1

2

2

2

3

24 
Table

$\underline{\text { Page }}$

1 Sensitivity of the Jerome Model 411 Mercury Vapor Analyzer

5

2 Precision of Mercury Determinations

6

3 Mercury Concentrations in Soils of 643-G Burial Ground

4 Statistical Summary of Field Measurements and Analyses of Adsorbed Mercury Concentrations

5 Statistical Distribution Symbols for Areal Maps 


\section{Figure}

1 Index Map, SRP Burial Ground 643-G

2 Site Locations, Area A, SRP Burial Ground 643-G

3 Site Locations, Area B, SRP Burial Ground 643-G

4 Site Locations, Area C, SRP Burial Ground 643-G

5 Site Locations and Adsorbed Mercury Concentrations, Swamp Area Adjacent to TNX Area

6 Distribution of Mercury Concentrations

7 Adsorbed Mercury Concentrations, Area A, SRP Burial Ground 643-G

8 Adsorbed Mercury Concentrations, Area B, SRP Burtal Ground 643-G

9 Adsorbed Mercury Concentrations, Area $\subset$, SRP Burial Ground 643-G 
Soil concentrations of adsorbed mercury at 999 sites at the Savannah River Plant (SRP) were determined by Microseeps Limited of Indianola, PA. The sites were in and around the 643-G Burial Ground, at the Savannah River Swamp adjacent to TNX Area, and at a background area. The Burial Ground was chosen as a test site because of a history of disposal of radioactive mercury there prior to 1968 (Horton, 1973). Extremely low traces of mercury have been detected in the water table beneath the Burial Ground (Oblath, 1985). Although the mercury concentrations at the majority of these sites are at background levels, several areas appear to be anomalously high. In particular, an area of large magnitude anomaly was found in the northwest part of the Burial Ground. Three other single point anomalies and several other areas of more subtle but consistently high values were also found. Several sites with anomalous mercury levels were found in an area of the Savannah River flood plain adjacent to TNX Area.

\section{Introduction}

Mercury occurs widely in nature and is associated with economically valuable mineral deposits. A trace element in many of the minerals of these ore deposits, mercury is also present in the metallic state due to oxidation of the host minerals. The relatively high volatility of mercury compared to the other heavy metals results in a uniquely high mobility and rapid migration in soils. Mercury is widely used as a pathfinder in geochemical exploration for base metal sulfides, gold, and silver. Pronounced local anomalies are often found directly above ore deposits; however, displacements of up to $2 \mathrm{~km}$ have been reported. The environmental fate of mercury vapor introduced into the atmosphere from natural sources such as active volcanoes and human activities such as ore refining and coal combustion has also stimulated investigation of the mercury cycle.

Fang (1978) and Landa (1978) investigated five Montana soils of differing characteristics to determine their efficiency and capacity to adsorb mercury vapor. Both the mineralogical makeup and organic matter content and type affected the adsorption capability of the soils. In both studies, dry soil samples were exposed to known concentrations of mercury vapor for measured lengths of time. Only a small fraction of the adsorbed mercury was in the oxidized mercuric form. The adsorbed mercury was not lost from the soils when maintained at air dryness and room temperature over a 16-day observation period. Solls maintalned at one-third bar moisture-tension for the same period showed a maximum loss of $5 \%$ of the total adsorbed mercury. There was no loss of mercury after the soils were placed in a vacuum desiccator for 24 $\mathrm{hr}$ or heated in an oven at $110^{\circ} \mathrm{C}$ for $2 \mathrm{hr}$. Major fractions (70-90\%) of the adsorbed mercury were desorbed from the soils at temperatures between 150 and $200{ }^{\circ} \mathrm{C}$. These data, coupled with the results of solvent extraction, suggest that 70 to $90 \%$ of the adsorbed mercury resides in the soils either in the form of organomercury complexes or as an inorganic complex.

The capacity of soils to retain mercury adsorbed as an organic or inorganic complex provides a sensitive mechanism for the accumulation of vaporous mercury flux in near-surface soils. The survey method used in this study was chosen because these mercury complexes can be decomposed by heat and because mercury will amalgamate to gold. 


\section{Sampling Areas}

Soil concentrations of adsorbed mercury were measured at 999 sites at the 643-G Burial Ground, at the Savannah River Swamp adjacent to TNX Area, and at a background area. The background samples (953-975) were collected at 23 sites in $Z$ Area, which is approximately 2.5 to $3 \mathrm{mi}$ northeast of the $643 . \mathrm{G}$ Burial Ground. This area was chosen because its soil type is similar to the Burial Ground's. Naturally adsorbed mercury levels could be expected to be similar to the Burial Ground, assuming no naturally occurrfing localized mineralization or man-made effects.

Samples were collected in three areas in and around the Burial Ground. The location of the sample sites, of known and probably burials of mercury, and of ground-water monitoring wells with elevated mercury levels are shown in Figures 1 through 4. For purposes of mapping and discussion, the data are broken into three areas: A, B, and C. Area A comprises 125 sites ( $1-93$ and 154-185) immediately south of the Burial Ground perimeter fence and a second traverse of 78 sites (471-548) immediately south of the main road (Figure 2).

Area B (Figure 3) is located at the west end of the Burial Ground. Four traverses in the south $(250-312)$, with spacing of $100 \mathrm{ft}$ by $50 \mathrm{ft}$, were sampled. Additional samples (313-470, 549-952) were collected in a 25 -ft center grid to the north. A line of samples (186-221) was collected along the
western fence of the Burial Ground.

Area $C$ is located in the east part of the Burial Ground and consists of seven lines of sample sites (94-153 and 222-249) with 50-ft sample spacing and 100-ft separations, as shown in Figure 4.

An additional 24 sites (976-999) were located in a swamp area in the Savannah River flood plain adjacent to TNX Area (Figure 5).

\section{Sample Collection}

A 5-cm diameter soil core was collected by driving a sharpened pipe to an approximate depth of $30 \mathrm{~cm}$ at each site. Each core was placed on a clean paper plate and allowed to air dry until it could be field sieved to 40 mesh $(0.5 \mathrm{~mm})$. Samples were placed in labeled bags and analyzed within $48 \mathrm{hr}$ of collection.

\section{Sample Analysis}

Mercury was desorbed from a $1-\mathrm{g}$ portion of the 40 -mesh fraction of each sample by heating the sample to $175{ }^{\circ} \mathrm{C}$ for $1 \mathrm{hr}$ in a $8 \mathrm{~mm}$ by $60 \mathrm{~mm}$ fused silica tube. The Inside bottom $25 \mathrm{~mm}$ of each silica tube was coated with a thin layer of gold that adsorbed the mercury released from the soil. Gold exhibits a maximum adsorptive capacity for mercury between 150 and $200{ }^{\circ} \mathrm{C}$. Thus, the mercury was concentrated in a form from which it could later be quickly released for analysis.

The silica tubes were cooled, the soil was discarded, and each sample was analyzed using a Jerome Model 411 Gold Film Mercury Analyzer. The mercury was released from the gold film in each silica tube by rapid heating to $550^{\circ} \mathrm{C}$ in 
a resistance heater. A flow of nitrogen swept the contents of each tube into that changes resistance as a function of adsorbed onto a calibrated gold film minimum detectable amount of merction of the amount of mercury adsorbed. The released from $1 \mathrm{~g}$ of soil corresponds is $0.5 \mathrm{ng}\left(0.5 \times 10^{-9} \mathrm{~g}\right)$, which if This level is approximately $10 \%$ of to a soll mercury content of $0.5 \mathrm{ppb}$. which previous exploration surveys the normal background soil mercury level,

Sensitivity of the Jerome Model 411 Gold Film Mercury Analyzer is reported to be 8 counts/ng and linear over its entire range. The instrument response and linearity were checked by injection of known amounts of air to $56.0 \mathrm{ng}$ was found to vapor at $20{ }^{\circ} \mathrm{C}$. Mean sensitivity over a range of 7.0 counts/ng. As shown in Table counts/ng with a standard deviation of 0.4 consistently over the range. 1 , the sensitivity increases slightly but two samples from sites 938 and 942 (Table 2). multiple runs were performed on cases are less than $10 \%$ of the mean value.

The mercury content of a garden soil from Indianola, PA, was determined prior to this survey. A 1 -Ib sample of soil was air dried and sieved to 40 mesh. Seventy-five gold-plated silica tubes were each filled with $1 \mathrm{~g}$ of th sieved soil and analyzed for adsorbed mercuryere each fllled with $1 \mathrm{~g}$ of this above. Mean adsorbed mercury content wascury using the method described deviation of $0.88 \mathrm{ppb}$ or $16 \%$ content was found to be $5.43 \mathrm{ppb}$ with a standard soil mercury content and the analytical varion reflects the variability of the

\section{Analytical Results}

The analytical results are presented in Table 3 . The data displayed a nonlinear distribution of mercury concentrations that approximated a lognormal distribution (Figure 6). The $\log$ mean is .8224 and the $10 \mathrm{~g}$-standard deviation is . 36 (Table 4). Table 5 shows the symbols used on the maps to represents an incremental change of Each successive change in symbol concentration.

The background data have an arithmetic mean of $7.6 \mathrm{ppb}$ and a standard deviation of $3.1 \mathrm{ppb}$. The distribution is near normal, with a variation coefficient of 0.41 . The range (min $=2 \mathrm{ppb}, \max -14 \mathrm{ppb}$ ) is approximately in geological sampling deviations. The threshold for anomalous concentrations deviations above the mean is well defined; however, use of 2 standard the data further suggests that non. Based on this definition, the range of background data set and that the anomalous concentrations exist in the represent the background levels in parameters could reasonably be expected to burial of mercury.

For the most part, the data in Area A (Figure 7) are at or near background values with the exception of two zones: the first at sites 22 and 23 and the second along the eastern half of the Burial Ground north of the anomalous based on the zones contain samples in excess of $23 \mathrm{ppb}$, which are and on the $\log$ normally $\log$ normally distributed data of Burial Ground $643-G$ these zones are located immediated data of the background area. Both of 

sites. The observed anomalous concentrations are considerably diminished, if
at all detectable, on the traverse south of the main road.

Anomalous zones (Figure 8) located in sections $\mathrm{C}-2$ and $\mathrm{D}-2$ of Area $\mathrm{B}$ contain several sites with mercury levels 3 to 4 log-standard deviations above the mean. These mercury levels are clearly not a part of the natural $F-1$. Sections E-2, E-3 point anomalies are noted in sections B-1, B-3, and adsorbed mercury in the 10 to and $E-5$ show a consistently elevated level of log-standard deviations above $\mathrm{ppb}$ range although the data are only 1 to 2 part of Area B do not reveal anye mean. The four traverses in the southern particular, do not suggest any source forle anomalous values and, in just south of the Burfal any source for the anomalous levels in section 7

No prominent anomalies are observed in Area C (Figure 9). Several consistently higher values are found in the northwest corner of this area, higher sites in sections A-29, A-30, B-29, B-30, C-29, and C-30. Several sections G-29 to 35 .

Samples collected from the Savannah River flood plain have concentrations of adsorbed mercury in the 4 to $200 \mathrm{ppb}$ range. The higher concentrations may be related to contamination but may just as well be related to the organic large area, the areal distribution pecause only 27 samples were taken from this for the observed mercury. 
TABLE 1

Sensitivity of the Jerome Model 411 Mercury Vapor Analyzer

Mercury

Injected

(ng)

Average Instrument

Instrument

Response (counts)

Sensitivity

7.0

53

(counts/ng)

14.0

114

7.6

28.0

234

8.1

42.0

356

8.4

56.0

482

8.5

8.6 
TABLE 2

Precision of Mercury Determinations (ppb)

Site 938

9.8

9.4

12.0

10.9

10.0

10.1

Mean $=10.4 \mathrm{ppb}$

$S D=0.94 \mathrm{ppb}$
Site 942

22.3

21.4

20.8

21.3

20.1

22.3

Mean - $21.4 \mathrm{ppb}$

$S D=0.86 \mathrm{ppb}$ 
TABLE 3

Mercury Concentrations (ppb) in Solls of 643-G Burlal Ground

\begin{tabular}{|c|c|c|c|c|c|c|c|}
\hline Site & Conc, & Site & Conc. & Site & Conc, & $\underline{\text { Site }}$ & Conc. \\
\hline 1 & 11 & 41 & 0 & 81 & 6 & 121 & 19 \\
\hline 2 & 8 & 42 & 2 & 82 & 8 & 122 & 9 \\
\hline 3 & 4 & 43 & 0 & 83 & 6 & 123 & 5 \\
\hline 4 & 5 & 44 & 3 & 84 & 5 & 124 & 4 \\
\hline 5 & 3 & 45 & 4 & 85 & 10 & 125 & 4 \\
\hline 6 & 4 & 46 & 3 & 86 & 13 & 126 & 7 \\
\hline 7 & 2 & 47 & 8 & 87 & 5 & 127 & 4 \\
\hline 8 & 2 & 48 & 4 & 88 & 5 & 128 & 5 \\
\hline 9 & 4 & 49 & 0 & 89 & 3 & 129 & 4 \\
\hline 10 & 3 & 50 & 4 & 90 & 16 & 130 & 6 \\
\hline 11 & 3 & 51 & 1 & 91 & 17 & 131 & 9 \\
\hline 12 & 2 & 52 & 1 & 92 & 28 & 132 & 13 \\
\hline 13 & 1 & 53 & 2 & 93 & 22 & 133 & 3 \\
\hline 14 & 2 & 54 & 2 & 94 & 10 & 134 & 16 \\
\hline 15 & 2 & 55 & 5 & 95 & 19 & 135 & 7 \\
\hline 16 & 2 & 56 & 7 & 96 & 20 & 136 & 7 \\
\hline 17 & 2 & 57 & 7 & 97 & 21 & 137 & 21 \\
\hline 18 & 2 & 58 & 8 & 98 & 3 & 138 & 28 \\
\hline 19 & 1 & 59 & 4 & 99 & 9 & 139 & 5 \\
\hline 20 & 0 & 60 & 3 & 100 & 26 & 140 & 4 \\
\hline 21 & 1 & 61 & 4 & 101 & 18 & 141 & 5 \\
\hline 22 & 26 & 62 & 5 & 102 & 13 & 142 & 8 \\
\hline 23 & 37 & 63 & 7 & 103 & 7 & 143 & 8 \\
\hline 24 & 4 & 64 & 6 & 104 & 10 & 144 & 17 \\
\hline 25 & 2 & 65 & 11 & 105 & 9 & 145 & 8 \\
\hline 26 & 1 & 66 & 16 & 106 & 2 & 146 & 9 \\
\hline 27 & 4 & 67 & 9 & 107 & 8 & 147 & 4 \\
\hline 28 & 0 & 68 & 30 & 108 & 21 & 148 & 4 \\
\hline 29 & 3 & 69 & 23 & 109 & 1 & 149 & 0 \\
\hline 30 & 1 & 70 & 8 & 110 & 5 & 150 & 3 \\
\hline 31 & 0 & 71 & 9 & 111 & 5 & 151 & 6 \\
\hline 32 & 0 & 72 & 5 & 112 & 8 & 152 & 4 \\
\hline 33 & 2 & 73 & 2 & 113 & 12 & 153 & 21 \\
\hline 34 & 10 & 74 & 3 & 114 & 5 & 154 & 26 \\
\hline 35 & 4 & 75 & 7 & 115 & 3 & 155 & 16 \\
\hline 36 & 0 & 76 & 16 & 116 & 9 & 156 & 40 \\
\hline 37 & 1 & 77 & 20 & 117 & 8 & 157 & 20 \\
\hline 38 & 0 & 78 & 36 & 118 & 5 & 158 & 27 \\
\hline 39 & 2 & 79 & 9 & 119 & 9 & 159 & 10 \\
\hline 40 & 2 & 80 & 6 & 120 & 3 & 160 & 5 \\
\hline
\end{tabular}


Table 3 (cont.)

\begin{tabular}{|c|c|c|c|c|c|c|c|}
\hline Site & Conc. & Site & Conc. & $\underline{\text { Site }}$ & Conc. & Site & Conc \\
\hline 161 & 21 & 212 & 10 & 263 & 5 & 314 & 4 \\
\hline 162 & 8 & 213 & 6 & 264 & 4 & 315 & 4 \\
\hline 163 & 8 & 214 & 4 & 265 & 6 & 316 & 3 \\
\hline 164 & 9 & 215 & 13 & 266 & 17 & 317 & 2 \\
\hline 165 & 14 & 216 & 3 & 267 & 9 & 318 & 5 \\
\hline 166 & 9 & 217 & 2 & 268 & 13 & 319 & 9 \\
\hline 167 & 4 & 218 & 2 & 269 & 5 & 320 & 4 \\
\hline 168 & 11 & 219 & 1 & 270 & 5 & 321 & 3 \\
\hline 169 & 8 & 220 & 1 & 271 & 4 & 322 & 1 \\
\hline 170 & 4 & 221 & 2 & 272 & 10 & 323 & 3 \\
\hline 171 & 8 & 222 & 14 & 273 & 4 & 324 & 8 \\
\hline 172 & 17 & 223 & 27 & 274 & 5 & 325 & 9 \\
\hline 173 & 14 & 224 & 21 & 275 & 4 & 326 & 9 \\
\hline 174 & 12 & 225 & 5 & 276 & 5 & 327 & -15 \\
\hline 175 & 7 & 226 & 11 & 277 & 2 & 328 & 7 \\
\hline 176 & 9 & 227 & 15 & 278 & 4 & 329 & 7 \\
\hline 177 & 6 & 228 & 23 & 279 & 2 & 330 & 3 \\
\hline 178 & 11 & 229 & 19 & 280 & 4 & 331 & 4 \\
\hline 179 & 22 & 230 & 5 & 281 & 3 & 332 & 6 \\
\hline 180 & 29 & 231 & 8 & 282 & 3 & 333 & 10 \\
\hline 181 & 9 & 232 & 4 & 283 & 5 & 334 & 3 \\
\hline 182 & 8 & 233 & 14 & 284 & 1 & 335 & 0 \\
\hline 183 & 7 & 234 & 26 & 285 & 6 & 336 & 5 \\
\hline 184 & 9 & 235 & 2 & 286 & 6 & 337 & 2 \\
\hline 185 & 10 & 236 & 7 & 287 & 3 & 338 & 4 \\
\hline 186 & 2 & 237 & 8 & 288 & 7 & 339 & 4 \\
\hline 187 & 2 & 238 & 8 & 289 & 1 & 340 & 5 \\
\hline 188 & 3 & 239 & 10 & 290 & 1 & 341 & 5 \\
\hline 189 & 5 & 240 & 1 & 291 & 3 & 342 & 673 \\
\hline 190 & 3 & 241 & 4 & 292 & 4 & 343 & 5 \\
\hline 191 & 26 & 242 & 3 & 293 & 2 & 344 & 3 \\
\hline 192 & 5 & 243 & 6 & 294 & 4 & 345 & 5 \\
\hline 193 & 5 & 244 & 5 & 295 & 5 & 346 & 5 \\
\hline 194 & 10 & 245 & 10 & 296 & 5 & 347 & 6 \\
\hline 195 & 6 & 246 & 11 & 297 & 7 & 348 & 7 \\
\hline 196 & 10 & 247 & 25 & 298 & 1 & 349 & 4 \\
\hline 197 & 4 & 248 & 7 & 299 & 3 & 350 & 2 \\
\hline 198 & 2 & 249 & 6 & 300 & 14 & 351 & 2 \\
\hline 199 & 1 & 250 & 8 & 301 & 9 & 352 & 2 \\
\hline 200 & 0 & 251 & 6 & 302 & 4 & 353 & 2 \\
\hline 201 & 2 & 252 & 12 & 303 & 3 & 354 & 5 \\
\hline 202 & 3 & 253 & 4 & 304 & 3 & 355 & 8 \\
\hline 203 & 3 & 254 & 8 & 305 & 28 & 356 & 5 \\
\hline 204 & 8 & 255 & 9 & 306 & 2 & 357 & 10 \\
\hline 205 & 9 & 256 & 5 & 307 & 1 & 358 & 6 \\
\hline 206 & 4 & 257 & 5 & 308 & 3 & 359 & 2 \\
\hline 207 & 5 & 258 & 3 & 309 & 2 & 360 & 8 \\
\hline 208 & 11 & 259 & 3 & 310 & 5 & 361 & 5 \\
\hline 209 & 8 & 260 & 3 & 311 & 2 & 362 & 6 \\
\hline 210 & 6 & 261 & 2 & 312 & 2 & 363 & 6 \\
\hline 211 & 2 & 262 & 13 & 313 & 3 & 364 & 8 \\
\hline
\end{tabular}


Table 3 (cont.)

\begin{tabular}{|c|c|}
\hline Sit & $\underline{\mathrm{C}_{C}}$ \\
\hline 365 & \\
\hline 366 & \\
\hline 367 & \\
\hline 368 & \\
\hline 369 & \\
\hline 370 & \\
\hline 371 & 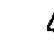 \\
\hline 372 & 4 \\
\hline 373 & 10 \\
\hline 374 & 3 \\
\hline 375 & 4 \\
\hline 376 & 4 \\
\hline 377 & 2 \\
\hline 378 & 3 \\
\hline 379 & 2 \\
\hline 380 & 1 \\
\hline 381 & 2 \\
\hline 382 & 5 \\
\hline 383 & 1 \\
\hline 384 & 22 \\
\hline 385 & 5 \\
\hline 386 & 9 \\
\hline 387 & 8 \\
\hline 388 & 13 \\
\hline 389 & 12 \\
\hline 390 & 9 \\
\hline 391 & 11 \\
\hline 392 & 14 \\
\hline 393 & 3 \\
\hline 394 & 2 \\
\hline 395 & 5 \\
\hline 396 & 4 \\
\hline 397 & 6 \\
\hline 398 & 8 \\
\hline 399 & 15 \\
\hline 400 & 11 \\
\hline 401 & 3 \\
\hline 402 & 13 \\
\hline 403 & 12 \\
\hline 404 & 5 \\
\hline 405 & 75 \\
\hline 406 & 5 \\
\hline 407 & 7 \\
\hline 408 & 17 \\
\hline 409 & 10 \\
\hline 410 & 137 \\
\hline 411 & 12 \\
\hline 412 & 10 \\
\hline 413 & 8 \\
\hline 414 & 9 \\
\hline 415 & 5 \\
\hline
\end{tabular}

\begin{tabular}{|c|c|}
\hline Site & \\
\hline 416 & \\
\hline 417 & \\
\hline 418 & 1 \\
\hline 419 & 1 \\
\hline 420 & 1 \\
\hline 421 & 1 \\
\hline 422 & 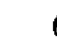 \\
\hline 423 & 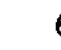 \\
\hline 424 & 9 \\
\hline 425 & 10 \\
\hline 426 & 12 \\
\hline 427 & 7 \\
\hline 428 & 24 \\
\hline 429 & 27 \\
\hline 430 & 9 \\
\hline 431 & $\epsilon$ \\
\hline 432 & $\epsilon$ \\
\hline 433 & 4 \\
\hline 434 & 5 \\
\hline 435 & 6 \\
\hline 436 & 7 \\
\hline 437 & 6 \\
\hline 438 & 4 \\
\hline 439 & 12 \\
\hline 440 & 7 \\
\hline 441 & 10 \\
\hline 442 & 1 \\
\hline 443 & 6 \\
\hline 444 & 18 \\
\hline 445 & 2 \\
\hline 446 & 9 \\
\hline 447 & 3 \\
\hline 448 & 3 \\
\hline 449 & 5 \\
\hline 450 & 3 \\
\hline 451 & 3 \\
\hline 452 & 4 \\
\hline 453 & 11 \\
\hline 454 & 4 \\
\hline 455 & 5 \\
\hline 456 & 7 \\
\hline 457 & 7 \\
\hline 458 & 2 \\
\hline 459 & 5 \\
\hline 460 & 2 \\
\hline 461 & 10 \\
\hline 462 & 9 \\
\hline 463 & 57 \\
\hline 464 & 13 \\
\hline 465 & 6 \\
\hline 466 & 12 \\
\hline
\end{tabular}

\begin{tabular}{|c|c|c|c|}
\hline Site & Conc. & Site & Cono \\
\hline 467 & 246 & 518 & 8 \\
\hline 468 & 7 & 519 & 1 \\
\hline 469 & 86 & 520 & 1 \\
\hline 470 & 436 & 521 & 2 \\
\hline 471 & 2 & 522 & 3 \\
\hline 472 & 2 & 523 & 5 \\
\hline 473 & 2 & 524 & 2 \\
\hline 474 & 0 & 525 & 1 \\
\hline 475 & 4 & 526 & 4 \\
\hline 476 & 2 & 527 & 3 \\
\hline 477 & 2 & 528 & 1 \\
\hline 478 & 3 & 529 & 2 \\
\hline 479 & 2 & 530 & 5 \\
\hline 480 & 1 & 531 & 11 \\
\hline 481 & 0 & 532 & 18 \\
\hline 482 & 1 & 533 & 8 \\
\hline 483 & 2 & 534 & 13 \\
\hline 484 & 2 & 535 & 5 \\
\hline 485 & 1 & 536 & 3 \\
\hline 486 & 1 & 537 & 9 \\
\hline 487 & 1 & 538 & 11 \\
\hline 488 & 1 & 539 & 5 \\
\hline 489 & 0 & 540 & 6 \\
\hline 490 & 1 & 541 & 3 \\
\hline 491 & 3 & 542 & 6 \\
\hline 492 & 1 & 543 & 4 \\
\hline 493 & 5 & 544 & 5 \\
\hline 494 & 5 & 545 & 3 \\
\hline 495 & 12 & 546 & 4 \\
\hline 496 & 0 & 547 & 6 \\
\hline 497 & 0 & 548 & 3 \\
\hline 498 & 1 & 549 & 124 \\
\hline 499 & 0 & 550 & 9 \\
\hline 500 & 0 & 551 & 11 \\
\hline 501 & 2 & 552 & 19 \\
\hline 502 & 2 & 553 & 12 \\
\hline 503 & 1 & 554 & 22 \\
\hline 504 & 3 & 555 & 10 \\
\hline 505 & 15 & 556 & 5 \\
\hline 506 & 11 & 557 & 8 \\
\hline 507 & 2 & 558 & 34 \\
\hline 508 & 1 & 559 & 16 \\
\hline 509 & 1 & 560 & 16 \\
\hline 510 & 2 & 561 & 14 \\
\hline 511 & 6 & 562 & 18 \\
\hline 512 & 3 & 563 & 16 \\
\hline 513 & 9 & 564 & 24 \\
\hline 514 & 5 & 565 & 11 \\
\hline 515 & 6 & 566 & 12 \\
\hline 516 & 6 & 567 & 7 \\
\hline 517 & 13 & 568 & 105 \\
\hline
\end{tabular}


Table 3 (cont.)

\begin{tabular}{|c|c|c|c|c|c|c|c|}
\hline Site & Conc & Site & Conc. & Site & Conc. & Site & Conc. \\
\hline 569 & 18 & 620 & 31 & 671 & 6 & 722 & 10 \\
\hline 570 & 11 & 621 & 17 & 672 & 7 & 723 & 13 \\
\hline 571 & 5 & 622 & 10 & 673 & 8 & 724 & 6 \\
\hline 572 & 7 & 623 & 13 & 674 & 6 & 725 & 8 \\
\hline 573 & 14 & 624 & 12 & 675 & 6 & 726 & 7 \\
\hline 574 & 10 & 625 & 9 & 676 & 7 & 727 & 9 \\
\hline 575 & 17 & 626 & 9 & 677 & 4 & 728 & 8 \\
\hline 576 & 20 & 627 & 7 & 678 & 7 & 729 & 8 \\
\hline 577 & 11 & 628 & 7 & 679 & 6 & 730 & 19 \\
\hline 578 & 23 & 629 & 7 & 680 & 7 & 731 & 13 \\
\hline 579 & 6 & 630 & 5 & 681 & 52 & 732 & 13 \\
\hline 580 & 8 & 631 & 10 & 682 & 13 & 733 & 19 \\
\hline 581 & 7 & 632 & 10 & 683 & 12 & 734 & 12 \\
\hline 582 & 6 & 633 & 6 & 684 & 15 & 735 & 7 \\
\hline 583 & 8 & 634 & 8 & 685 & 12 & 736 & 7 \\
\hline 584 & 172 & 635 & 6 & 686 & 38 & 737 & 7 \\
\hline 585 & 13 & 636 & 6 & 687 & 11 & 738 & 3 \\
\hline 586 & 97 & 637 & 9 & 688 & 7 & 739 & 8 \\
\hline 587 & 9 & 638 & 5 & 689 & 10 & 740 & 11 \\
\hline 588 & 13 & 639 & 6 & 690 & 5 & 741 & 7 \\
\hline 589 & 9 & 640 & 8 & 691 & 14 & 742 & 3 \\
\hline 590 & 17 & 641 & 4 & 692 & 27 & 743 & 5 \\
\hline 591 & 17 & 642 & 7 & 693 & 12 & 744 & 4 \\
\hline 592 & 10 & 643 & 9 & 694 & 22 & 745 & 4 \\
\hline 593 & 11 & 644 & 6 & 695 & 13 & 746 & 9 \\
\hline 594 & 8 & 645 & 6 & 696 & 9 & 747 & 11 \\
\hline 595 & 8 & 646 & 4 & 697 & 11 & 748 & 8 \\
\hline 596 & 4 & 647 & 5 & 698 & 10 & 749 & 5 \\
\hline 597 & 5 & 648 & 7 & 699 & 8 & 750 & 4 \\
\hline 598 & 6 & 649 & 8 & 700 & 25 & 751 & 5 \\
\hline 599 & 7 & 650 & 8 & 701 & 11 & 752 & 9 \\
\hline 600 & 14 & 651 & 9 & 702 & 3 & 753 & 7 \\
\hline 601 & 12 & 652 & 4 & 703 & 14 & 754 & 12 \\
\hline 602 & 14 & 653 & 3 & 704 & 13 & 755 & 10 \\
\hline 603 & 14 & 654 & 8 & 705 & 12 & 756 & 6 \\
\hline 604 & 25 & 655 & 7 & 706 & 6 & 757 & 9 \\
\hline 605 & 11 & 656 & 2 & 707 & 8 & 758 & 16 \\
\hline 606 & 21 & 657 & 3 & 708 & 8 & 759 & 13 \\
\hline 607 & 16 & 658 & 3 & 709 & 9 & 760 & 12 \\
\hline 608 & 20 & 659 & 6 & 710 & 12 & 761 & 11 \\
\hline 609 & 7 & 660 & 5 & 711 & 8 & 762 & 14 \\
\hline 610 & 6 & 661 & 6 & 712 & 6 & 763 & 2 \\
\hline 611 & 6 & 662 & 5 & 713 & 4 & 764 & 6 \\
\hline 612 & 6 & 663 & 2 & 714 & 4 & 765 & 10 \\
\hline 613 & 13 & 664 & 2 & 715 & 9 & 766 & 11 \\
\hline 614 & 8 & 665 & 9 & 716 & 7 & 767 & 19 \\
\hline 615 & 54 & 666 & 6 & 717 & 8 & 768 & 10 \\
\hline 616 & 14 & 667 & 4 & 718 & 12 & 769 & 6 \\
\hline 617 & 14 & 668 & 6 & 719 & 14 & 770 & 9 \\
\hline 618 & 18 & 669 & 2 & 720 & 19 & 771 & 15 \\
\hline 619 & 11 & 670 & 20 & 721 & 19 & 772 & 10 \\
\hline
\end{tabular}


Table 3 (cont.)

\begin{tabular}{|c|c|c|c|c|c|c|c|}
\hline Site & Conc. & Site & Conc. & Site & Conc. & Site & Conc \\
\hline 773 & 5 & 824 & 11 & 875 & 13 & 926 & 5 \\
\hline 774 & 10 & 825 & 10 & 876 & 8 & 927 & 5 \\
\hline 775 & 13 & 826 & 18 & 877 & 4 & 928 & 4 \\
\hline 776 & 7 & 827 & 11 & 878 & 6 & 929 & 2 \\
\hline 777 & 9 & 828 & 29 & 879 & 3 & 930 & 5 \\
\hline 778 & 13 & 829 & 4 & 880 & 4 & 931 & 6 \\
\hline 779 & 13 & 830 & 8 & 881 & 5 & 932 & 3 \\
\hline 780 & 7 & 831 & 14 & 882 & 5 & 933 & 5 \\
\hline 781 & 13 & 832 & 6 & 883 & 13 & 934 & 6 \\
\hline 782 & 10 & 833 & 7 & 884 & 16 & 935 & 5 \\
\hline 783 & 12 & 834 & 8 & 885 & 12 & 936 & 2 \\
\hline 784 & 14 & 835 & 10 & 886 & 8 & 937 & 4 \\
\hline 785 & 12 & 836 & 7 & 887 & 3 & 938 & 10 \\
\hline 786 & 7 & 837 & 10 & 888 & 3 & 939 & 3 \\
\hline 787 & 9 & 838 & 7 & 889 & 4 & 940 & 13 \\
\hline 788 & 6 & 839 & 2 & 890 & 3 & 941 & 21 \\
\hline 789 & 28 & 840 & 7 & 891 & 3 & 942 & 22 \\
\hline 790 & 17 & 841 & 11 & 892 & 7 & 943 & 3 \\
\hline 791 & 8 & 842 & 7 & 893 & 5 & 944 & 3 \\
\hline 792 & 7 & 843 & 16 & 894 & 13 & 945 & 4 \\
\hline 793 & 5 & 844 & 10 & 895 & 11 & 946 & 7 \\
\hline 794 & 10 & 845 & 9 & 896 & 18 & 947 & 4 \\
\hline 795 & 14 & 846 & 8 & 897 & 4 & 948 & 5 \\
\hline 796 & 6 & 847 & 13 & 898 & 11 & 949 & 4 \\
\hline 797 & 5 & 848 & 13 & 899 & 5 & 950 & 7 \\
\hline 798 & 14 & 849 & 8 & 900 & 6 & 951 & 3 \\
\hline 799 & 15 & 850 & 10 & 901 & 18 & 952 & 15 \\
\hline 800 & 4 & 851 & 5 & 902 & 7 & 953 & 6 \\
\hline 801 & 15 & 852 & 10 & 903 & 5 & 954 & 11 \\
\hline 802 & 13 & 853 & 9 & 904 & 25 & 955 & 14 \\
\hline 803 & 6 & 854 & 9 & 905 & 2 & 956 & 8 \\
\hline 804 & 12 & 855 & 12 & 906 & 8 & 957 & 8 \\
\hline 805 & 8 & 856 & 11 & 907 & 7 & 958 & 6 \\
\hline 806 & 9 & 857 & 8 & 908 & 4 & 959 & 8 \\
\hline 807 & 19 & 858 & 7 & 909 & 12 & 960 & 7 \\
\hline 808 & 8 & 859 & 7 & 910 & 7 & 961 & 7 \\
\hline 809 & 9 & 860 & 11 & 911 & 10 & 962 & 4 \\
\hline 810 & 5 & 861 & 3 & 912 & 7 & 963 & 2 \\
\hline 811 & 12 & 862 & 6 & 913 & 16 & 964 & 2 \\
\hline 812 & 11 & 863 & 1 & 914 & 5 & 965 & 6 \\
\hline 813 & 3 & 864 & 9 & 915 & 6 & 966 & 10 \\
\hline 814 & 10 & 865 & 5 & 916 & 3 & 967 & 6 \\
\hline 815 & 18 & 866 & 8 & 917 & 1 & 968 & 12 \\
\hline 816 & 5 & 867 & 5 & 918 & 7 & 969 & 10 \\
\hline 817 & 5 & 868 & 9 & 919 & 5 & 970 & 9 \\
\hline 818 & 8 & 869 & 5 & 920 & 5 & 971 & 6 \\
\hline 819 & 16 & 870 & 4 & 921 & 5 & 972 & 11 \\
\hline 820 & 5 & 871 & 3 & 922 & 9 & 973 & 4 \\
\hline 821 & 11 & 872 & 2 & 923 & 5 & 974 & 6 \\
\hline 822 & 9 & 873 & 11 & 924 & 2 & 975 & 11 \\
\hline 823 & 10 & 874 & 13 & 925 & 7 & 976 & 30 \\
\hline
\end{tabular}


Table 3 (cont.)

\section{Site Conc.}

\begin{tabular}{rr}
977 & 23 \\
978 & 202 \\
979 & 40 \\
980 & 78 \\
981 & 49 \\
982 & 21 \\
983 & 34 \\
984 & 28 \\
985 & 14 \\
986 & 15 \\
987 & 4 \\
988 & 5 \\
989 & 4 \\
990 & 7 \\
991 & 4 \\
992 & 10 \\
993 & 7 \\
994 & 3 \\
995 & 13 \\
996 & 15 \\
997 & 48 \\
998 & 69 \\
999 & 19 \\
\hline
\end{tabular}

Note: The values in this table were truncated. 


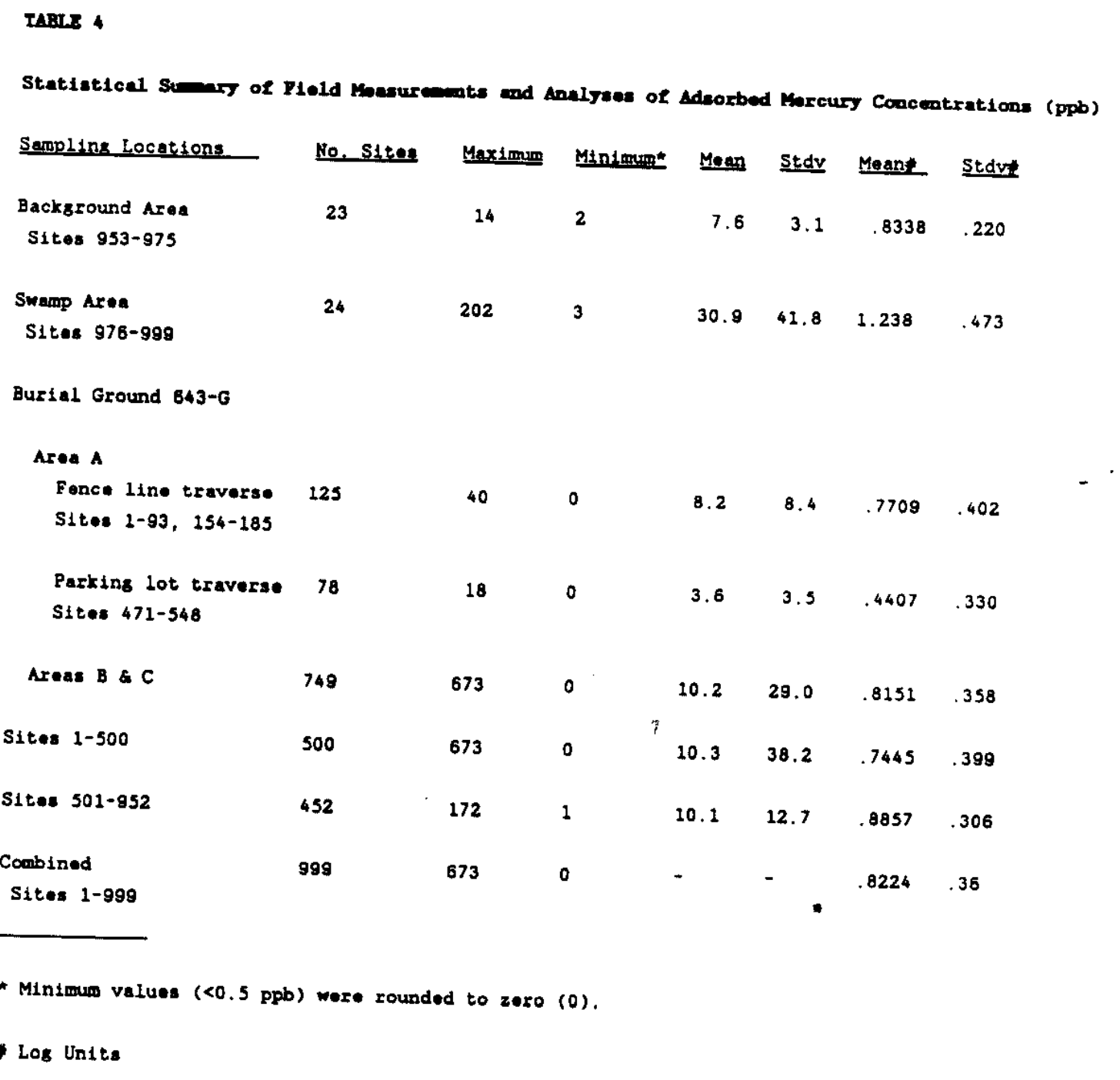


TABLE 5

Stat1stical Distribution Symbols for Areal Maps

Symbol Hg Conc. (ppb) No. Sites $\begin{aligned} & \text { Percentile } \\ & \text { Ranget }\end{aligned}$ Standard Dev.

南>120.8 $\quad 7 \quad>99.3 \quad>2.082$

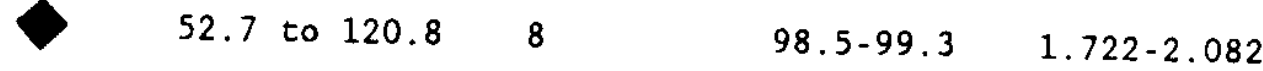

- $\quad 23.0$ to $52.7 \quad 35 \quad 95.0-98.5 \quad 1.362-1.722$

- 10.0 to $23.0 \quad 214 \quad 73.6-95.0 \quad 1.002-1.362$

O 4.4 to $10.0 \quad 434 \quad 30.1 .73 .6 \quad 0.642-1.002$

$0 \quad 1.9$ to $4.4 \quad 240 \quad 6.1-30.1 \quad 0.282-0.642$

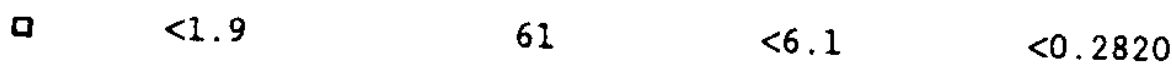

Note: $\log$ Mean $=.8224 ;$ Log standard deviation -.36 .

* Percentile assumes accumulation from the smallest to the largest. 

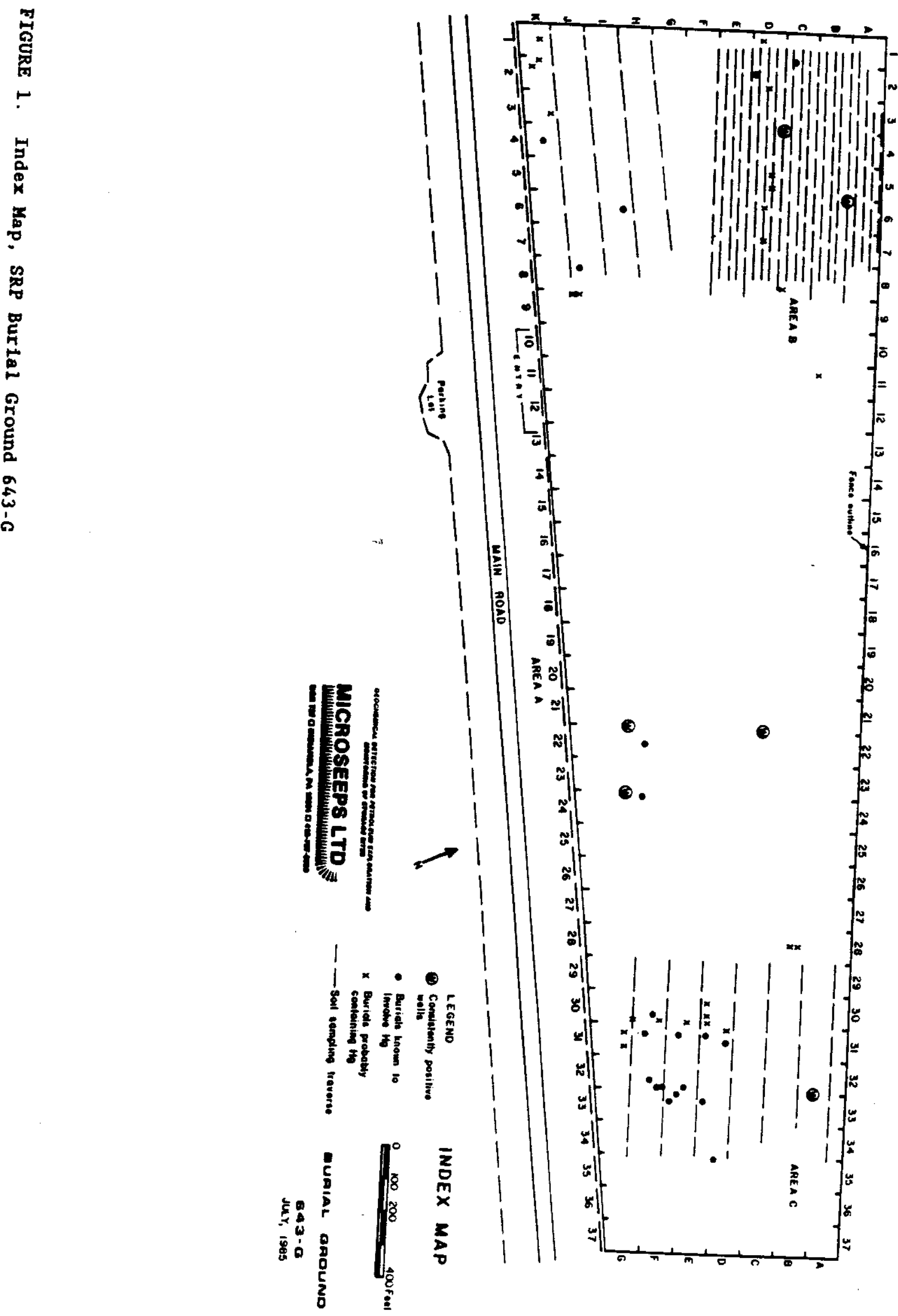


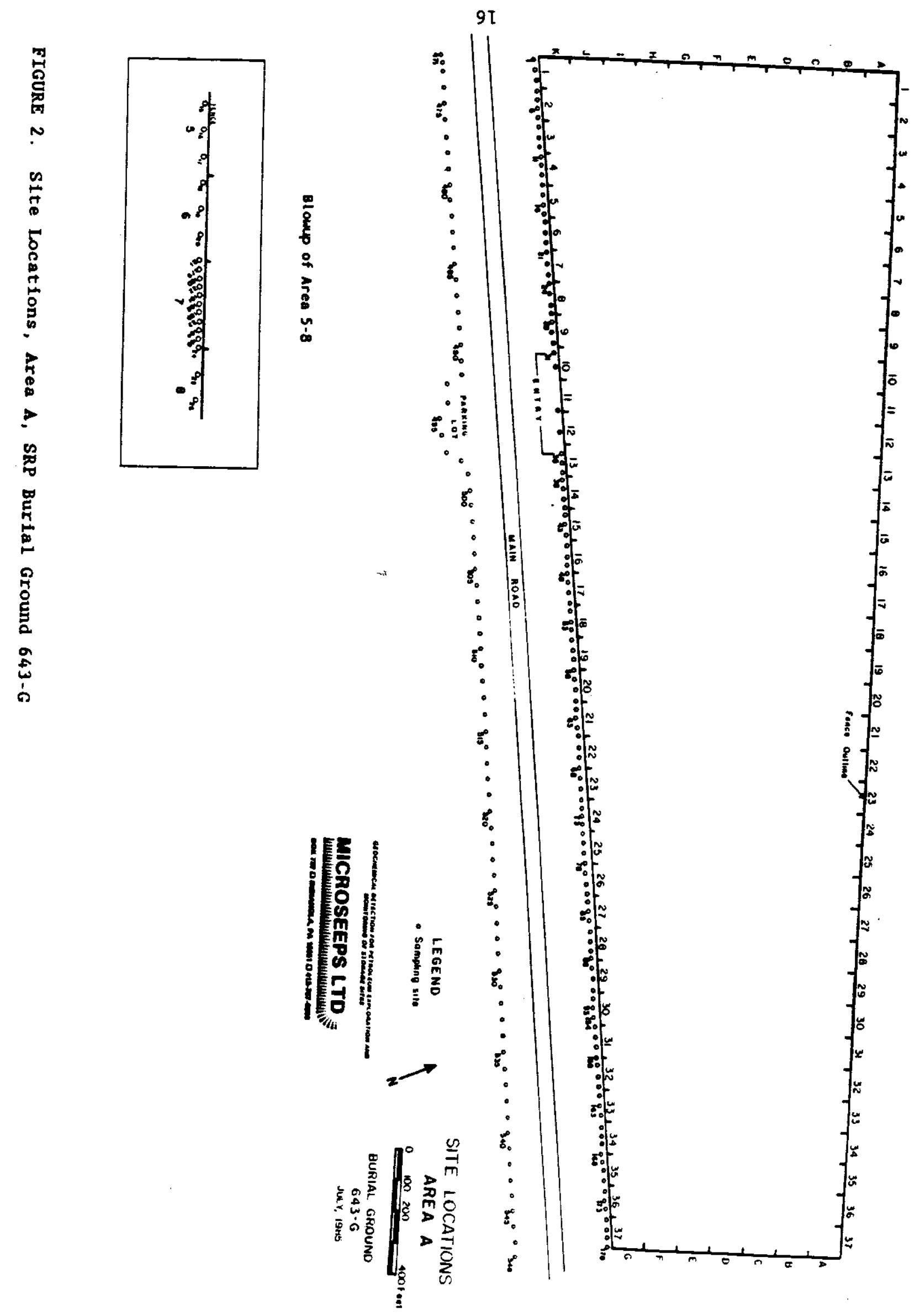




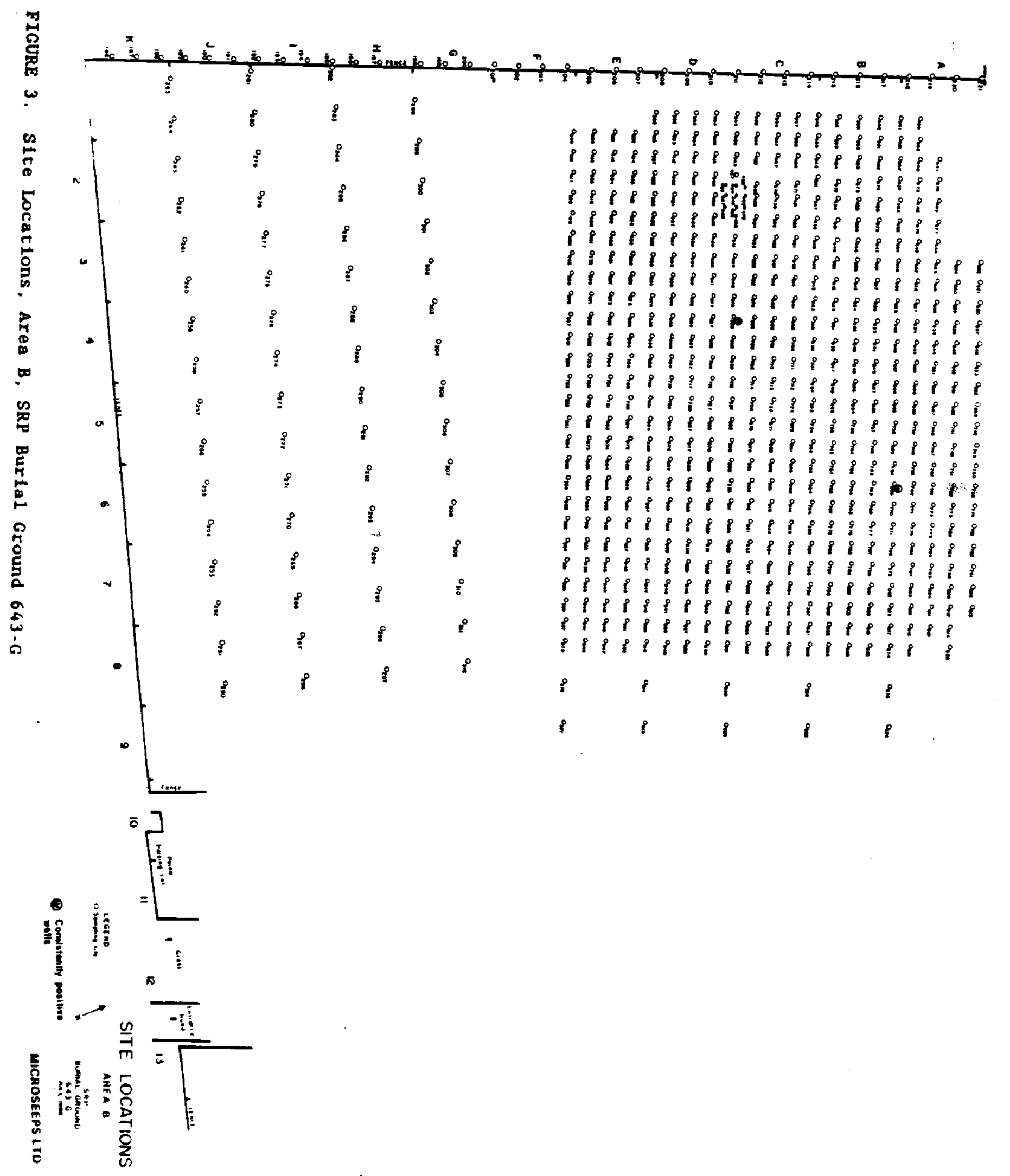


匀

$\stackrel{0}{\pi}$

$d$

5

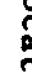

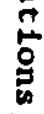

7

3.

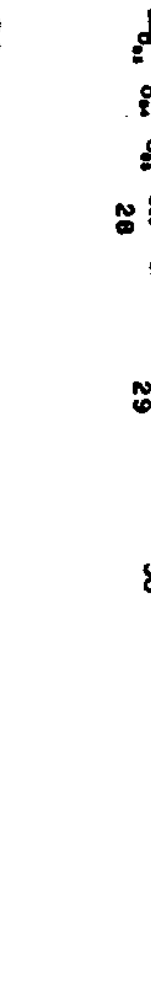




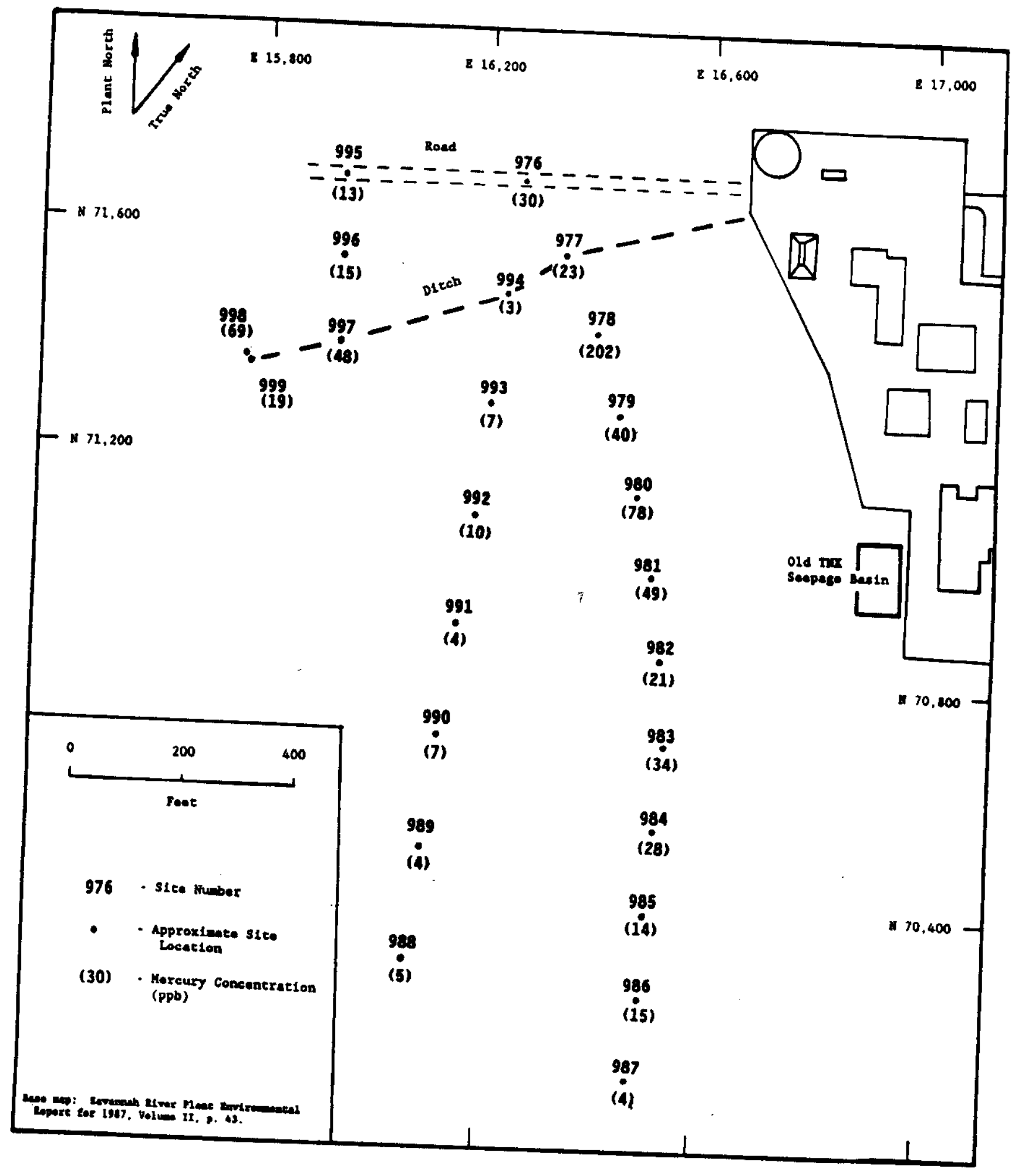

FIGURE 5. Site Locations and Adsorbed Mercury Concentrations, Swamp Area
Adjacent to TNX Area 


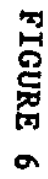

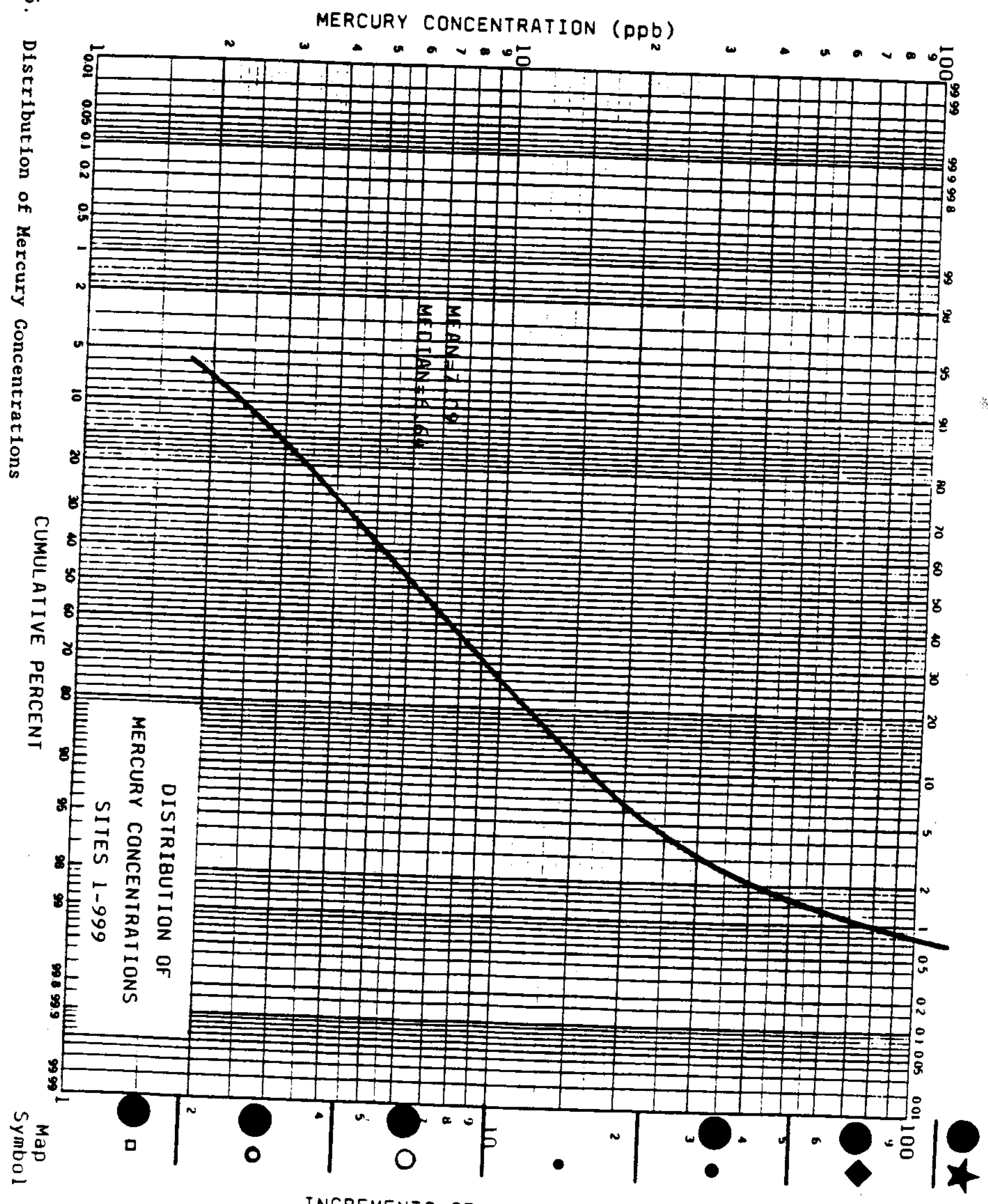

INCREMENTS OF LOG STANDARD DEVIATION 

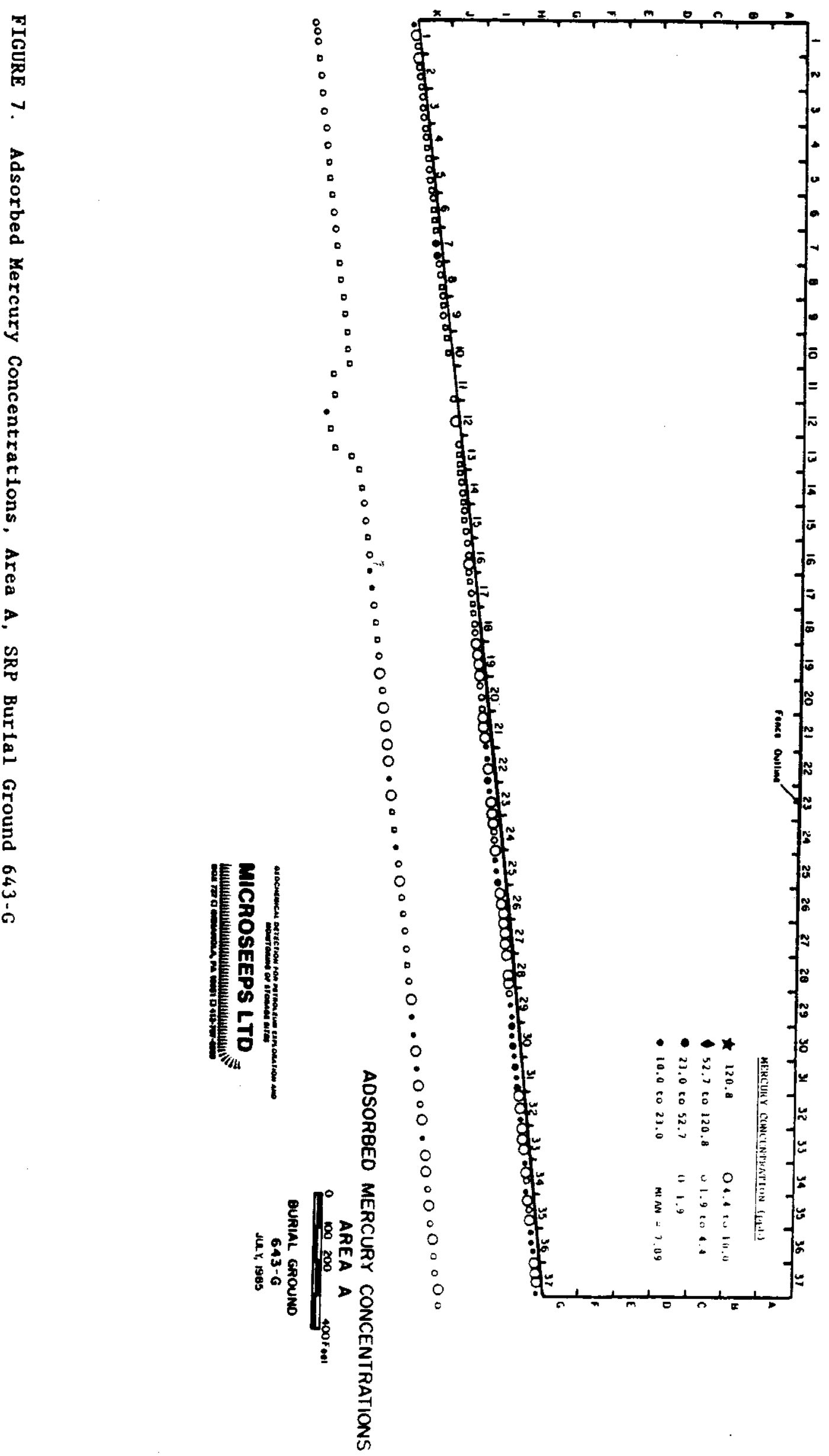


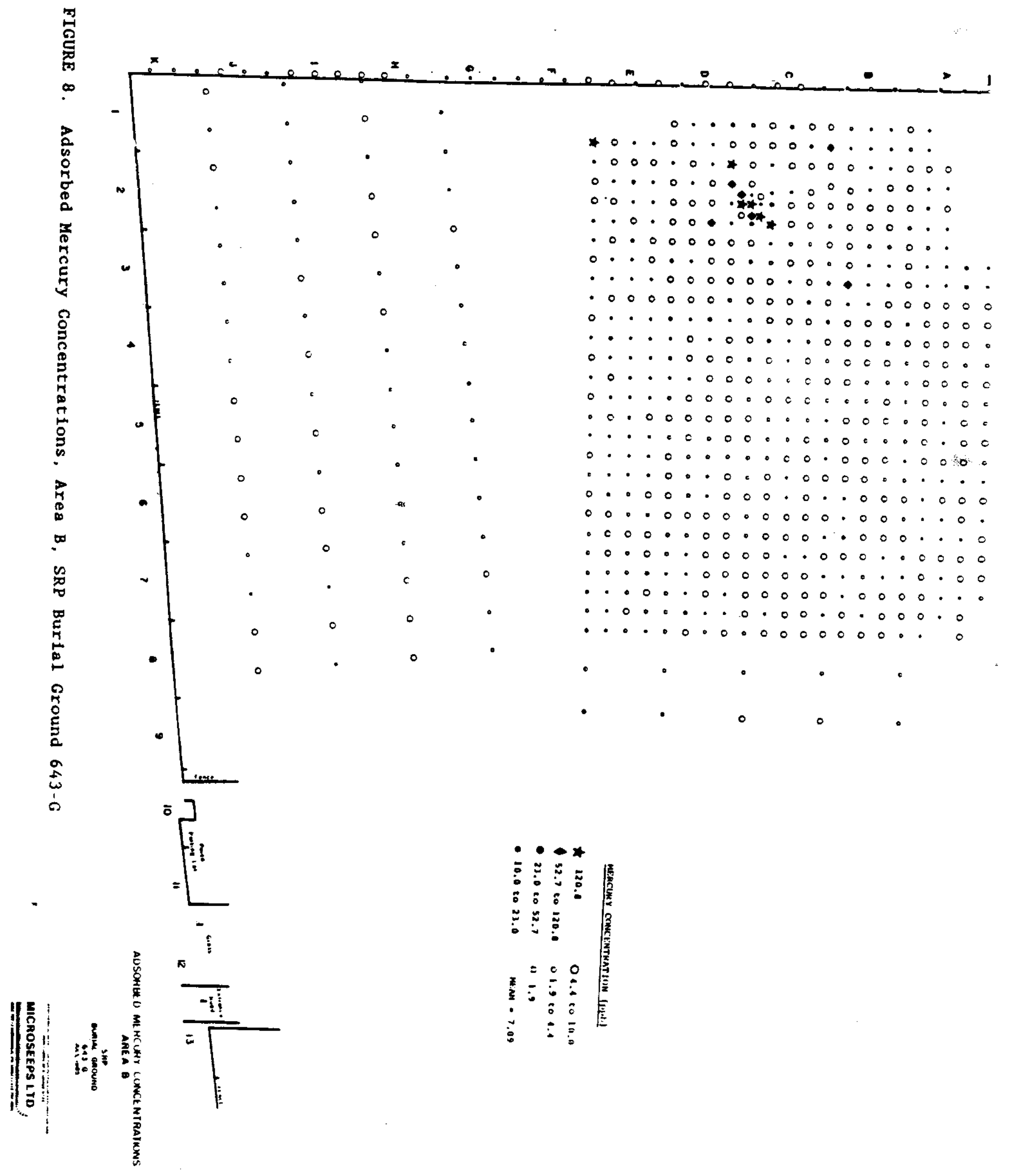




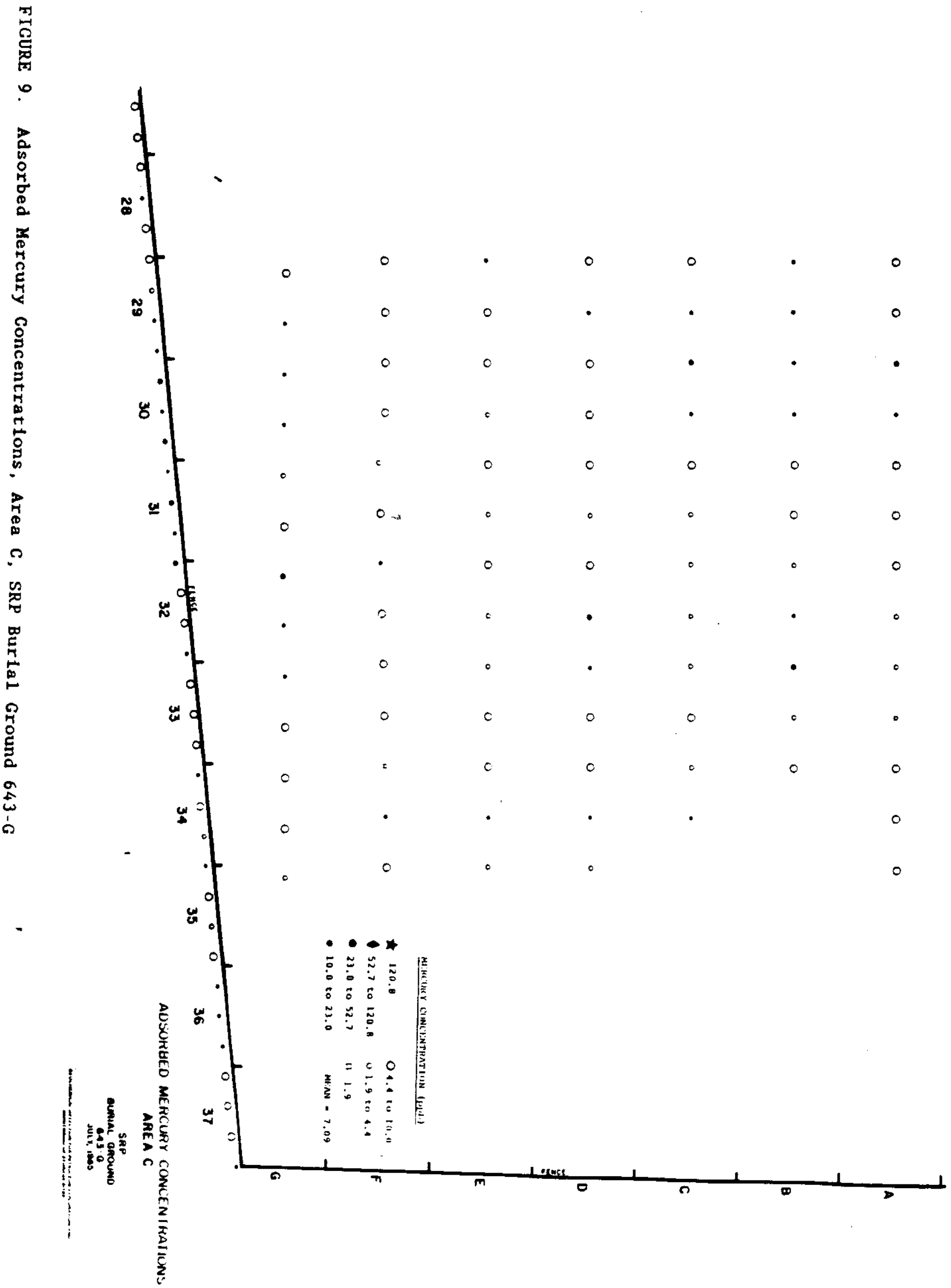




\section{References}

Fang, S. C., 1978. "Sorption and Transformation of Mercury-Vapor by Dry
Soil," Environmental Sclence Technology, $12(3): 285-88$.

Horton, J. H., 1973. Mercury in the 643-G Bur1al Ground, DPST-73-480, E. I. du Pont de Nemours and Company, Savannah River Laboratory, Aiken, S.C.

Landa, E. R., 1978. "Retention of Metallic Mercury Vapor by Soils," Geochimica et Cosmochimica Acta, 42:1407-11.

Oblath, S. B., 1985. 1984 Monitoring of Mercury in the Ground Water at $643-G$ and 643-7G, DPST-85-407, E. I. du Pont de Nemours and Company, Savannah
River Laboratory, Aiken, S.C. 